\title{
A hybrid model predictive control for traffic flow stabilization and pollution reduction of freeways
}

\author{
Alfréd Csikós, István Varga and Katalin M. Hangos \\ A. Csikós and K.M. Hangos are with Systems and Control Laboratory, Institute for Computer Science and Control, \\ Hungarian Academy of Sciences, Kende utca 13-17, 1111, Budapest, Hungary (e-mail: csikos.alfred@sztaki.mta.hu, \\ hangos@mta.sztaki.hu). I. Varga is with Budapest University of Technology and Economics, Department of Control for \\ Transportation and Vehicle Systems, Stoczek utca 2., 1111, Budapest, Hungary. (e-mail: ivarga@mail.bme.hu).
}

January 4, 2018

\begin{abstract}
In this work a control system is developed and analyzed for the suppression of moving jamwaves and the reduction of pollutant concentrations near motorways. The system is based on the second-order macroscopic freeway traffic model METANET, joined by an emission dispersion model, introduced in a previous work of the authors. For the control tasks dedicated controllers are designed, both using the nonlinear model predictive control method. The control objectives require a distinction in the utilized control measures, thus different controllers are designed and used in predefined control modes. The first mode of the controller is responsible for keeping pollutant concentrations below prescribed limits under stable conditions. The second mode of the controller is working in case of a shockwave threat, aiming for traffic stabilization. Between the control modes switching is based on an appropriate rule set that satisfies the stability of the controlled system. The hybrid controller structure is realized by a finite automata. A complex case study is presented for the evaluation of the suggested controller.
\end{abstract}

\section{Introduction}

The dispersion of vehicular emissions is a significant environmental problem: the pollution of certain exhaust gases (i.e. $\mathrm{CO}, \mathrm{HC}$ and $\mathrm{NO}_{\mathrm{x}}$ ) is responsible for serious health issues. Therefore, reduction of pollutant concentrations at inhabited areas near motorways is of vital importance in modern transport engineering. In our research, we aim to develop a motorway control system for a combined task: in addition to traffic stabilization, the controller should be capable of keeping pollutant concentrations below limits in the vicinity of motorways.

The effect of traffic management systems on vehicular emissions have been investigated both in motorways and urban networks by several authors in the past decades, see Szeto et al. (2012). The exploitation of vehicular emission models in traffic engineering research can be realized on different levels. The first level is the use of ITS (Intelligent transportation systems) data for offline or online emission modeling. Offline modeling efforts imply data-based analysis of the relationship between flow conditions and emission. Smit et al. (2008) examine the effect of mean speed distributions on traffic emission inventories. In Liu et al. (2011), the correlation of traffic patterns and emission data are analyzed, aiming to understand the relationship between daily flow level patterns and emissions. In Gokhale (2012), the environmental effect of synchronised flow patterns at intersections is analyzed. Fontes et al. (2015) compare the Eulerian and Lagrangian simulation approaches and their effect on the fidelity of emission inventories.

Online emission modeling results focus on the use of real-time data and this approach serves to establish control strategies including emission performances as control criteria. Chen et al. 
(2014) analyze the substitution of vehicle trajectory data to emission models resulting in average emission factors, normalized for vehicle unit. Chang et al. (2013) propose a bottom-up vehicle emission model to estimate $\mathrm{CO}_{2}$ emissions using real-time data. The proposed method uses loop detectors and floating car data to express average emission factors of local fleet compositions. Ryu et al. (2013) suggest a method to use probe vehicle data (or floating car data) to express average emissions emerging at link units. Zegeye et al. (2013) apply macroscopic traffic variables for the cycle-variable model VT-Micro (Rakha et al. (2004)) to express real-time average emission factors of traffic.

On the middle-level, emission models are used for the analysis of the environmental impact of ITS tools. In Carolien et al. (2007) a microscopic simulation platform is developed for the analysis of traffic control measures. Papers of Ma et al. (2014) and Jazcilevich et al. (2015) propose systematic assessment methodologies of ITS solutions, including their impact on emissions. Analyses are carried out regarding the influence of traffic intensity, signal coordination schemes and signal parameters on the gaseous emissions in urban networks by Coensel et al. (2012) and Gori et al. (2015). The effect of speed limit control is compared to the effect of road pricing in Yang et al. (2012) in terms of traffic performance and emission.

The incorporation of emission models to the control design is the highest level of use of emission models in traffic engineering. This was first done by Zegeye et al. (2009). The work of Mahmod et al. (2013) gives a comparative analysis of ITS measures (i.e. demand control, restriction of vehicle classes, and speed limit control) for a single intersection. The paper points out that constraining traffic demand clearly reduces emissions, while the reduction of speed limits lead to the increase of emission of certain pollutants. A special traffic assignment problem, minimizing network emissions is addressed by Long et al. (2016). Based on the Link Transmission Model, the problem is solved by means of mixed integer linear programming. A variable speed limit control scheme is designed to reduce emission factors on freeways by Liu et al. (2012). The data transferred by vehicle-to-vehicle (V2V) technologies can be best exploited for cycle variable or higher level emission models, an interesting control approach in this field is presented by Alsabaan et al. (2013).

It has to be pointed out, that although several approaches have been suggested to use emission models in traffic control analysis and synthesis, none of these approaches define a macroscopic description for the spatiotemporal distribution of emissions. This description, based on the average-speed vehicle emission modeling framework is derived by Csikós \& Varga (2012).

All the above works focus on emission modeling, expressed in terms of emission factors. For the pollutant behaviour of exhaust gases, roadside dispersion models can be applied. Shorshani et al. (2015) examine dispersion under calm wind conditions, following the Gaussian plume approach. A control-oriented dispersion model is presented by Zegeye et al. (2011) with a gridbased approach. The pollution is considered as a soft constraint, and a multi-objective control approach for traffic performance improvement and pollution reduction is proposed.

Inclusion of dispersion dynamics into control design is not straightforward. Handling pollutant concentrations as soft constraints in multi-objective design offers a general approach, however, in case of extreme demands and traffic jams it may lead to suboptimal solutions. On the other hand, topological attributes of the polluted areas need to be incorporated into control design as well. These points can be most effectively solved by separating the considered control problems, namely: i) stabilizing traffic flow; ii) keeping pollutant concentrations under specified limits under stable traffic conditions. Therefore, in the current paper, instead of a multi-objective control design, a hybrid approach is presented: separate controllers are developed for the above given control problems. Between the two controllers switching is realized by a supervisor controller, formalized as a finite automata that applies a set of rules that provides stability of the switching mechanism.

Our work relies on the dispersion model proposed in Csikós et al. (2015), where a sensitivity 
analysis of the model is also carried out supporting a preliminary statement of control objectives for pollution reduction. By integrating the emission dispersion model to the second-order motorway model METANET (Papageorgiou et al. (1990)), a joint system is obtained for which the hybrid controller is designed. Ramp metering and variable speed limit (VSL) control input values are optimized by means of the nonlinear model predictive control (NMPC) technique, see in Grune \& Pannek (2011). For the evaluation of the suggested controller, a complex case study is presented, in which performances of the control modes and the switching behaviour are analyzed.

The paper is structured as follows: after the introductory section, in Section 2 the macroscopic traffic and emission models are summarized alongside the emission dispersion model. Following an overview of the system model, control design is detailed in Section 3. The controller performance is analyzed in a case study, presented in Section 4. The computational properties of the proposed control system are discussed in Section 5. Finally, conclusions are drawn.

\section{Methods and tools}

Motorway traffic is most commonly described by macroscopic models using aggregated variables, which are bivariate functions of space and time. This distributed parameter system approach (DPS, see Hangos \& Cameron (2001)) is extended to the modeling of motorway traffic emissions as well: i.e. emission is stated as a variable of both space and time, expressed as a function of the macroscopic traffic variables. In this section the modeling aspects are summarized for traffic flow, traffic emissions and the dispersion of exhaust pollution.

\subsection{Motorway traffic model}

The considered traffic system is a motorway stretch divided to $N_{s}$ segments of similar length. ${ }^{1}$ Control of the system is realized by using metered ramps $r_{i}(k)$ and variable speed limits $v s l_{i}(k)$, $i=1, \ldots, N_{s}$. Traffic density $\rho_{i}(k)$, mean speed $v_{i}(k)$ and ramp queue $l_{i}(k)$ for discrete step $k$ and segment $i=1, \ldots, N_{s}$ are modeled by the second-order model METANET, see Papageorgiou et al. (1990). The dynamic equations of the model are given in Section 2.4, alongside the detailed description of model variables. In this section, the focus is on the modeling of variable speed limits (VSL).

In the METANET model, the equilibrium speed equation is defined as follows:

$$
V\left(\rho_{i}(k)\right)=v_{f r e e} \exp \left(-\frac{1}{a}\left(\frac{\rho_{i}(k)}{\rho_{c r}}\right)^{a}\right)
$$

where parameters $v_{f r e e}, a, \rho_{c r}$ are constant parameters, usually given as functions of the speed limit value. Here, a slight modification of the equilibrium speed equation of METANET is carried out based on the following assumption: by using variable speed limits, the free flow speed parameter $v_{\text {free }}$ is altered to $v s l_{i}$. Thus, the equilibrium speed in case of VSL control becomes a fraction of the uncontrolled speed function (1):

$$
V\left(\rho_{i}(k)\right)=v s l_{i}(k) \exp \left(-\frac{1}{a}\left(\frac{\rho_{i}(k)}{\rho_{c r}} \cdot\right)^{a}\right)
$$

Thus, the only result of speed control is the reduced capacity of the road. Nevertheless, certain VSL models (i.e. Hegyi et al. (2002) and Papageorgiou et al. (2008)) describe a slight increase in the critical density, thus the extension of the stable domain of the system as well, meaning an

\footnotetext{
${ }^{1}$ Segment lengths $L_{i}, i=1, \ldots, N_{s}$ may be chosen arbitrarily as long as the numerical stability condition of Courant et al. (1928) is satisfied for given sampling time $T$, i.e. $v_{f r e e} \frac{T}{L_{i}}<1, i=1, \ldots, N_{s}$. The free flow speed $v_{\text {free }}$ is equal to the highest velocity that a particle (i.e. a vehicle) in the flow may carry.
} 
additional stabilizing effect of VSL control. However, this effect has not yet been validated and is neglected in present work. The applied speed limit model has been successfully implemented for a real network case study in Goatin et al. (2016).

\subsection{Macroscopic traffic emission modeling}

For describing the vehicular emissions, several models exist in the microscopic level (e.g. MOVES, (see Chamberlin et al. (2011)), COPERT, (see Ntziachristos et al. (2000)), VERSIT, (see Smit et al. (2007))). The pollution of motorway traffic, however, needs to be described similarly to the traffic flow, i.e., as a function of space and time. The framework applied here relies on the microscopic vehicular emission models and extends them to the macroscopic level by means of macroscopic flow data. A distinction is made in the pollution modelling of the main network elements (i.e. the main lane and the ramp queue), due to the different types of available flow data. While the main lane is characterized by traffic density and mean speed measurements, measurement of on-ramps is narrowed to queue length data, without the information of ramp flow speed.

Main lane In this case, for vehicular emissions, the average-speed based modeling approach is adopted. Average-speed emission models give the emission factor ef of a single vehicle in units of $[\mathrm{g} / \mathrm{km}]$ as a function of the vehicle speed. For a quantitative analysis of average-speed emission modelling with different traffic models see Zhu et al. (2013).

In the followings, the macroscopic description of main lane emission is summarized (for a detailed introduction of the concept, the reader is referred to Csikós \& Varga (2012)). We assume that the vehicle composition is homogeneous and constant in time and its emission factor for pollutant $p$ is represented by $e f^{p}$. Then, the spatiotemporal distribution of traffic emission in the continuous domain of space $x$ and time $t$ for pollutant $p$ is given as follows :

$$
\varepsilon_{\text {main }}^{p}(x, t)=e f^{p}(v(x, t)) \rho(x, t) v(x, t),
$$

where continuous variables $\rho(x, t)$ and $v(x, t)$ denote the traffic density and mean speed at $(x, t)$, respectively. The variable $\varepsilon^{p}(x, t)$ is obtained in units $\left[\frac{\mathrm{vehg}}{\mathrm{km} \times \mathrm{h}}\right]$. Total emission $E^{p}$ of pollutant $p$ in unit [veh g] over a finite spatiotemporal domain $\Delta x \times \Delta t$ is given by the integral

$$
E_{\text {main }, \Delta x \times \Delta t}^{p}=\int_{\Delta t} \int_{\Delta x} \varepsilon^{p}(x, t) d x d t .
$$

Assuming that the average speed of traffic flow represents the speed of individual vehicles over a domain $L_{i} \times T$, definition (3) can be extended to discrete space and time, by averaging as in eq. (4). For a discrete segment $i$ of length $L_{i}$ in discrete sample step $k$ of duration $T$, the spatiotemporal distribution of emission of pollutant $p$ is given as:

$$
\varepsilon_{i, \text { main }}^{p}(k)=e f^{p}\left(v_{i}(k)\right) \rho_{i}(k) v_{i}(k),
$$

in units $\left[\frac{\mathrm{vehg}}{\mathrm{km} \times \mathrm{h}}\right]$. By eq. (5), the spatiotemporal distribution of traffic emission is formalized as a bivariate function of traffic density and traffic mean speed.

Ramp queue The queued vehicles of the ramp are considered to be idling. For these vehicles, instead of the emission factor, a time specific variable is used: the emission rate $e^{p}$, given in units $[\mathrm{g} / \mathrm{h}]$. In the macroscopic aspect, the emission of the on-ramp queue of segment $i$ is considered as belonging to the segment. In the discrete framework it is given as follows:

$$
\varepsilon_{i, r a m p}^{p}(k)=l_{i}(k) e^{p}(k) \frac{1}{L_{i}} \text {. }
$$


The spatiotemporal distribution of the emission caused by motorway traffic is given as the sum of eqs. (5) and (6), obtained in units $\left[\frac{\mathrm{vehg}}{\mathrm{km} \times \mathrm{h}}\right]$ :

$$
\varepsilon_{i}^{p}(k)=\varepsilon_{i, \text { main }}^{p}(k)+\varepsilon_{i, \text { ramp }}^{p}(k) .
$$

\subsection{Emission dispersion modeling}

The dispersion model aims at modeling the evolving concentration of the pollutants with local effects $\left(\mathrm{CO}, \mathrm{HC}, \mathrm{NO}_{\mathrm{X}}\right)$ at the boundary of inhabited areas near motorways. Here, a short summary of the modeling assumptions and the obtained state dynamics of emission dispersion is given. The derivation of the model is summarized in Appendix A. For a detailed derivation and analysis of the model the reader is referred to Csikós et al. (2015).

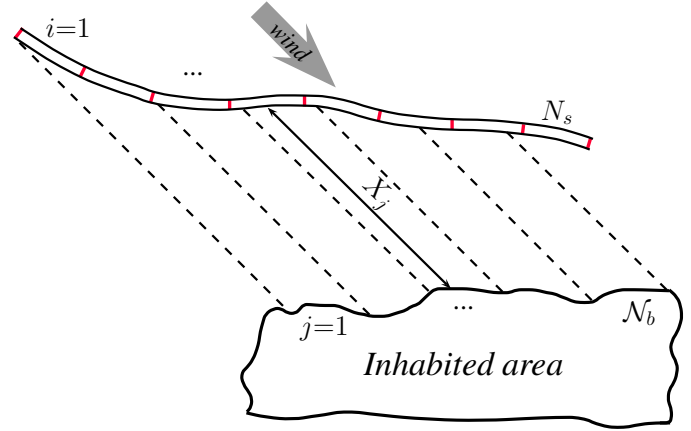

(a) Balance volumes near a motorway

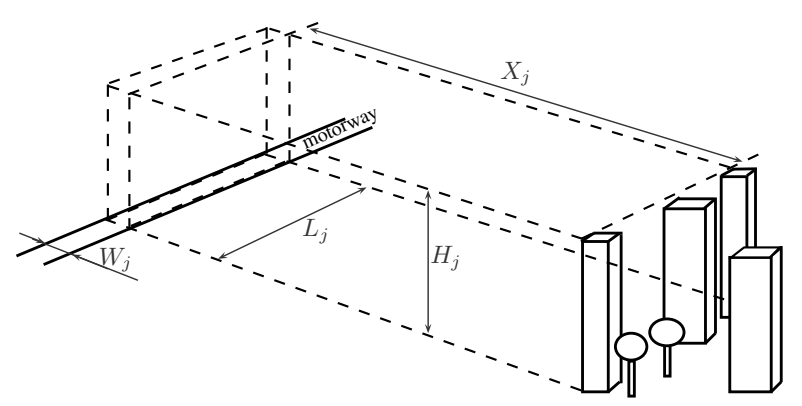

(b) Balance volume parameters

Figure 1: Topological layout of the dispersion model

The layout of the modeling problem is illustrated in Fig. 1a: the area between the road and the inhabited area is divided to $\mathcal{N}_{b}$ constant cross-section channels of equal width, parallel to the wind direction. (The dimensions $\{L, X, H, W\}_{j}$ of flow channel $j$ are illustrated in Fig. 1b). The process of emission dispersion is considered a distributed parameter system according to Hangos \& Cameron (2001), modeled separately for each flow channel. The output of the model are the pollutant concentrations at the boundary of the inhabited area.

The geometric parameters of a flow channel are illustrated in Fig. 1. The following modeling assumptions are adapted:

- Constant wind direction and changing wind speed are supposed, describing the effect of prevailing winds.

- The pollution is ideally mixed over the cross section of the flow channels. Only axial dispersion of pollution present through the channels.

- Plug flow is modeled within each flow channel $j, j=1, \ldots, \mathcal{N}_{b}$ (see Fig. 1a).

- The flow channels are considered as balance volumes in which the law of mass conservation (see Hangos \& Cameron (2001)) is formalized.

- The flow channels are parallel to the wind direction and are of equal width.

- For the boundary of the channels, the excitation is calculated by using the macroscopic description of traffic emission (see Section 2.2). 
The discrete dynamics of concentration in balance volume $j$ for time step $k$ is stated as:

$$
c_{j}^{p}(k+1)=c_{j}^{p}(k)+T\left(w(k) \frac{c_{j}^{p, 0}(k)-c_{j}^{p}(k)}{X_{j}}-\lambda_{j}(k) c_{j}^{p}(k)\right),
$$

where dissolution rate $\lambda$ is calculated as given in Appendix A, see eq. (23).

The initial condition is given by

$$
c_{j}^{p}(0)=0, \forall x_{j} \in\left[0, X_{j}\right],
$$

whereas boundary condition $c_{j}^{p, 0}(k)$ is stated based on the macroscopic emissions (7) emerging at the root of the balance volume:

$$
c_{j}^{p, 0}(k)=T \frac{\sum_{j, i=1}^{N_{j, i}} E_{j, i}^{p}(k) L_{j, i}}{H_{j} L_{j} W_{j}} .
$$

Remark 1 The number of the flow channels depends on the wind direction. The width of a flow channel is suggested to be chosen such that on average a flow channel is fed by one motorway segment. In most cases, however, due to the curvature of the motorway, $N_{s} \neq \mathcal{N}_{b}$, i.e. the number of motorway segments and that of the flow channels may differ (see Fig. 1a). The curvature also implies that a single balance volume may be fed by a changing number of motorway segments. In the boundary condition (9) the index $j, i=1, \ldots, N_{j, i}$ gives the motorway segments that feed balances volume $j$ along $L_{j, i}$ of their segment length $L_{i}$. For each balance volume $j, \sum_{j, i=1}^{N_{j, i}} L_{j, i}=L_{j}$ holds.

\subsection{The joint traffic - emission dispersion system}

The above proposed dispersion model is attached to the second-order traffic process model METANET. The two dynamics are coupled by the boundary conditions of the concentration dynamics, formalized as the function of traffic variables. The original state-space of the traffic model is extended by the outlined emission dispersion dynamics, the size $\mathcal{N}_{b}$ of which depends on the wind direction and topographic characteristics.

The state equation of the model is outlined in eq. (10) below. For easier readability, it is given in the form $x(k+1)=f(x(k))+g(x(k), u(k))+h(x(k), d(k))$, where $x(k)$ is the state, $u(k)$ is the input and $d(k)$ is the disturbance variable at time instant $k$. The state equations hence contain separately the solely state-dependent terms from the terms that contain the control inputs and the disturbance variables. 


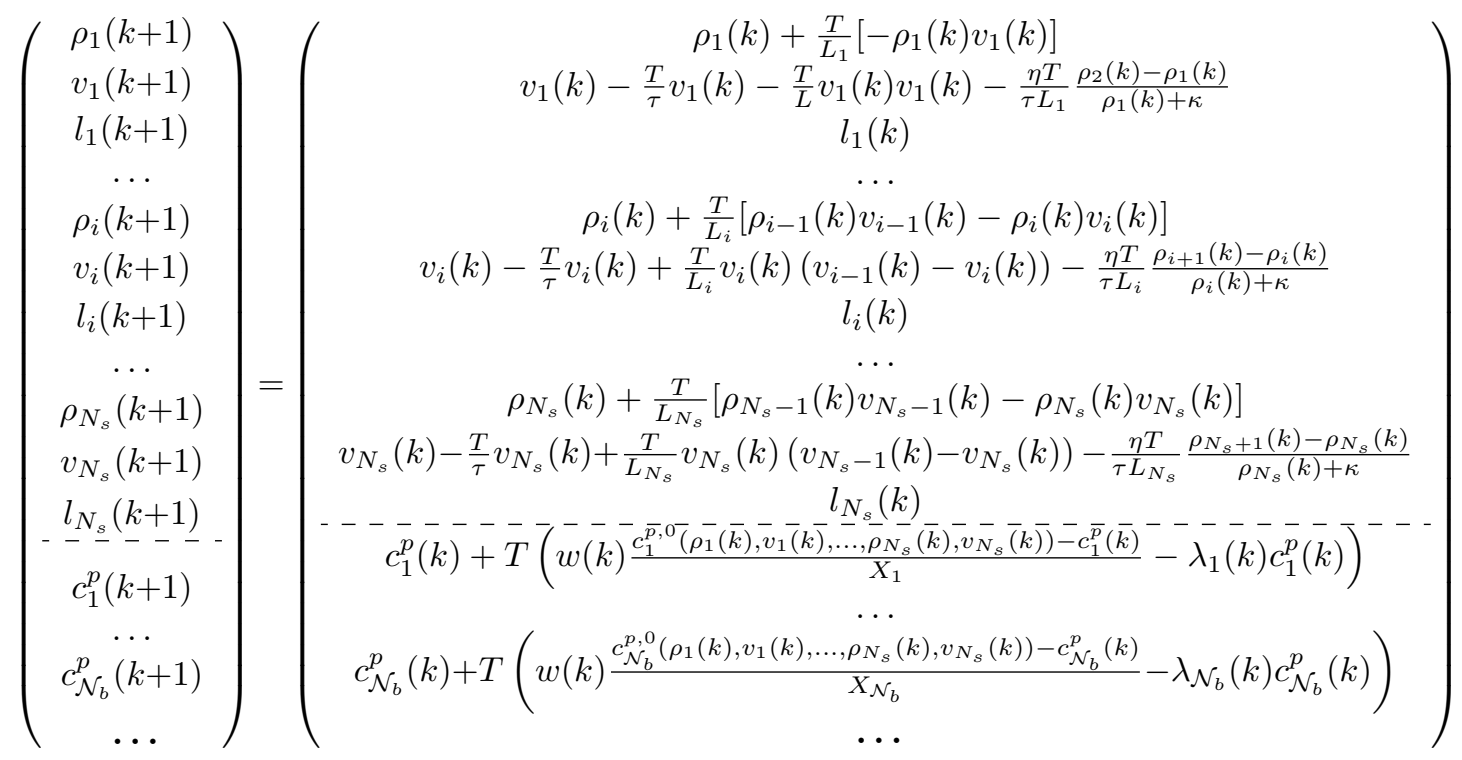

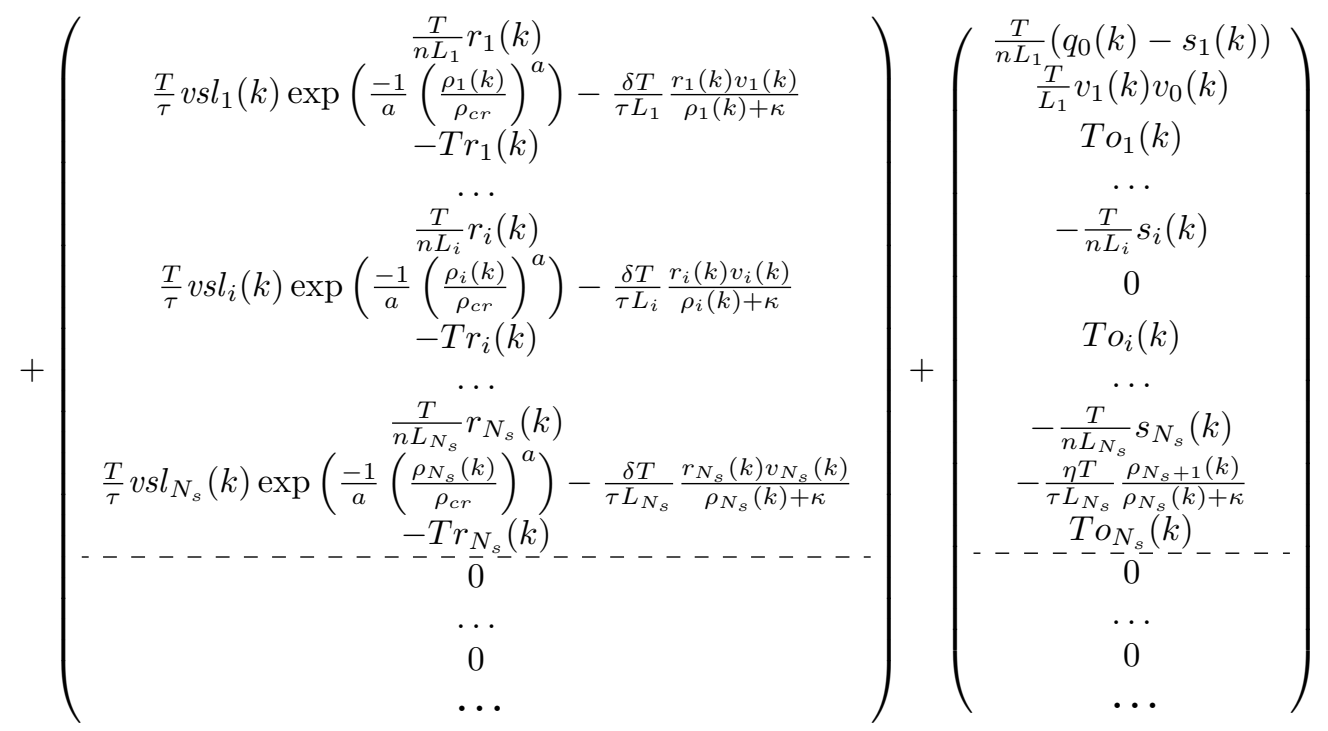

Above the dashed line of eq. (10) the dynamic equations of the model METANET are given for each segment $i$ through the triplet of states $\left(\rho_{i}(k), v_{i}(k), l_{i}(k)\right)$. Traffic states are governed by conservation laws: for the traffic density $\rho_{i}$ and ramp queue $l_{i}$, the conservation of vehicles; whereas for the mean speed $v_{i}$ the conservation of momentum is modeled. The last block below the dashed line involves the assumed conservation dynamics for the pollution within balance volumes. The notation "..." indicates that the block has to be repeated for all considered pollutants $p \in\left\{\mathrm{CO}, \mathrm{HC}, \mathrm{NO}_{X}\right\}$.

The state vector is hence obtained as follows:

$$
x(k)=\left[\rho_{1}(k), v_{1}(k), l_{1}(k), \ldots, \rho_{N_{s}}(k), v_{N_{s}}(k), l_{N_{s}}(k), c_{1}^{p}(k), \ldots, c_{\mathcal{N}_{b}}^{p}(k), \ldots\right]^{T} \in \mathbb{R}^{3 N_{s}+3 \mathcal{N}_{b}} .
$$

The input vector is in the form:

$$
u(k)=\left[r_{1}(k), \ldots, r_{N_{s}}(k), v s l_{1}(k), \ldots, v s l_{N_{s}}(k)\right]^{T} \in \mathbb{R}^{2 N_{s}} .
$$

It is important to note that all control inputs are not necessarily present, the highest dimension of the control input $\left(2 N_{s}\right)$ is stated.

For the motorway model the boundary variables of the network are considered disturbances, i.e., the upstream flow $q_{0}$, mean speed of the upstream traffic $v_{0}$, on-ramp demands 
$\left(o_{i}, i=1, \ldots, N_{s}\right)$, off-ramp flows $\left(s_{i}, i=1, \ldots, N_{s}\right.$ and downstream density $\left(\rho_{N_{s}+1}\right)$. The dispersion dynamics are influenced by disturbance variable wind speed $w(k)$ only. The measured disturbances of the joint system are collected in the following vector:

$$
d(k)=\left[q_{0}(k), v_{0}(k), \rho_{N_{s}+1}(k), w(k), o_{1}(k), \ldots, o_{N_{s}}(k), s_{1}(k), \ldots, s_{N_{s}}(k)\right]^{T} \in \mathbb{R}^{2 N_{s}+4} .
$$

Measured outputs are given in the vector $y(k)$ :

$$
y(k)=\left[\rho_{1}(k), v_{1}(k), l_{1}(k), \ldots, \rho_{N_{s}}(k), v_{N_{s}}(k), l_{N_{s}}(k)\right]^{T} \in \mathbb{R}^{3 N_{s}} .
$$

The METANET model is characterized by $\tau, \eta, \delta, \kappa$ constant parameters. Furthermore, dimensional parameters of the balance volumes, i.e., $\{X, L, W, H\}_{j}$ constitute of the constant parameters of the dispersion system.

\section{Control design}

The sensitivity analysis of the dispersion model (presented in Csikós et al. (2013b)) showed that concentration regulation as a control objective is in conflict with conventional traffic stabilizing interventions which do not necessarily improve concentration levels. While ramp metering can reduce the emission production and thus the emerging concentrations, VSL control leads to higher emissions within a finite spatiotemporal domain through the increased traffic densities, and thus higher pollutant concentrations are reached. Under stable conditions, however, VSL control is not needed. Thus, a feasible aim is to reduce pollutant concentrations below legislation limits, under stable conditions only, using the ramp metering and no VSL. Unstable traffic conditions require a different approach. Shockwaves (in our analysis, backward propagating jamwaves) can reach such extent and magnitude that the ramp metering by itself is not capable of suppressing them: in this case, both VSL and ramp metering inputs are needed.

The difference in the applied control inputs for the control aims requires two different control modes, each having individual control design. The switching between the control modes has to be based on an appropriate rule set that satisfies the stability of the controlled system. In what follows, a hybrid control structure is suggested for the above problem. After the statement of control objectives, a hybrid controller and its control modes are proposed.

\subsection{Control objectives}

In the followings, preliminary control objectives are stated, focusing on the main goal of each control mode. In Sections 3.3 and 3.4, the applied cost functions are stated, containing additional terms to improve controller performance.

- In case of stable traffic conditions, (under the critical density) the control aims to keep the concentrations below legislation limit $c_{\text {limit }}^{p}$, using the ramp metering only (for a detailed analysis of controller setup, see Csikós et al. (2014)). State constraints are defined as follows:

$$
c_{j}^{p}(k) \leq c_{\text {limit }}^{p} .
$$

The cost function of the control should be specified so that the ramp queues are minimized.

- In case of unstable traffic conditions (e.g. as a result of a shockwave), concentration constraints are neglected and a traffic stabilizing controller is used. This controller uses both ramp metering and variable speed limits. For a detailed description of the shockwave suppression control design see Csikós et al. (2013a).

On both levels, optimal input is designed by a nonlinear model predictive control (NMPC) design (see Grune \& Pannek (2011)), however with different cost functions and constraints.

The proposed two-mode controller has to handle both the uncongested and congested situation, aiming for the appropriate control objective. The key feature of the controller is the 
switching rule set that is able to recognize the neccessity of switching in both directions based on the system states with a stable operation, i.e., no oscillation of the modes emerges. The controllers are embedded in a hybrid automata model framework that also involves the switching logic, outlined in Section 3.2. The controller modes are detailed in Sections 3.3 and 3.4.

\subsection{Control system structure}

In this section the operation of the two-mode controller is outlined by the formal description of the control system structure.

A system is considered a hybrid system if it combines subsystems that are continuous in behavior (having a continuous set of states) with discrete event subsystems (having discrete states only). In our case, the subsystem with continuous behavior is the motorway traffic system, and the discrete event system is the subsystem that assigns the control mode (control for concentration/congestion). The switching rule is thus designed through the discrete event system specification. The discrete event system is represented by a finite automata model, which is designed based on both analytical considerations and heuristic design.

Following the conventional formal description of Hangos \& Cameron (2001), a hybrid system is described by the elements:

$$
H A=\left(D_{H A}, C_{H A}\right),
$$

where $H A$ denotes the hybrid automata model, which is composed of the discrete event system $D_{H A}$ and the continuous system $C_{H A}$.

The discrete event system is a finite automata model in the form of

$$
D_{H A}=\left(Q_{H A}, \Sigma_{H A}, \delta_{H A}\right),
$$

where $Q_{H A}$ is a set of different operational regions of the continuous state system. In our case,

$$
Q_{H A}=\{\text { congested traffic', 'free flow' }\} .
$$

$\Sigma_{H A}$ is the set of input elements of the finite automata that consists of autonomous plant events:

$$
\Sigma_{H A}=\{\text { 'forming congestion','dissolving congestion', 'saturation' }\} .
$$

$\delta_{H A}$ describes the possible operational regions that occur as a result of the state-event pairs:

$$
\delta_{H A}: Q_{H A} \times \Sigma_{H A} \mapsto Q_{H A} .
$$

In our case,

$$
\begin{aligned}
& \delta_{H A}(\text { 'free flow', 'forming congestion')='congested traffic', } \\
& \delta_{H A}(\text { 'congested traffic', 'forming congestion')='congested traffic', } \\
& \delta_{H A}(\text { 'congested traffic', 'dissolving congestion')='free flow', } \\
& \delta_{H A}(\text { 'free flow', 'dissolving congestion') }=\text { 'free flow', } \\
& \delta_{H A}(\text { 'congested traffic', 'saturation')='congested traffic', } \\
& \delta_{H A}(\text { 'free flow', 'saturation') }=\text { 'free flow'. }
\end{aligned}
$$

The state transition diagram of the discrete event system $D_{H A}$ is shown in Fig. 2.

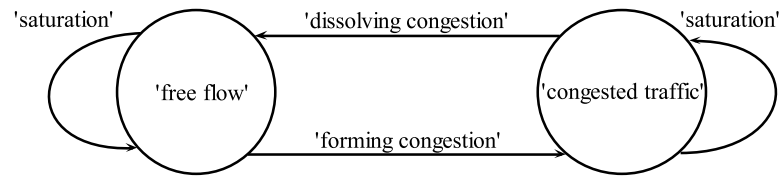

Figure 2: State transition diagram 
The continuous state system $C_{H A}$ of the structure is the system described earlier in Section 2.4. The nonlinear state dynamics are described by eq. (10). where measured outputs can take values within the set $y(k) \in Y_{p} \subseteq R^{3 N_{s}}$.

The events that form the set $\Sigma_{H A}$ are determined as follows. For the thresholds, values around the critical density are chosen. Furthermore, they are tuned considering the hysteresis effect in the traffic dynamics (see Yuan et al. (2017)): the choice of unequal threshold values leads to the exclusion of the oscillation and ensures the stability of the switching control. The threshold values are chosen based on manual tuning following a set of test runs.

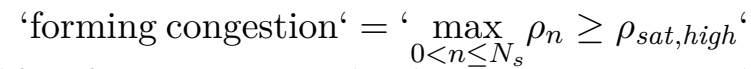

$$
\begin{aligned}
& \text { 'dissolving congestion' }={ }^{6} \max _{0<n \leq N_{s}} \rho_{n} \leq \rho_{\text {sat, low }}{ }^{6} \\
& \text { 'saturation' }=\text { ' } \rho_{\text {sat }, \text { low }} \leq \max _{0<n \leq N_{s}} \rho_{n} \leq \rho_{\text {sat, high }} \text { ' }
\end{aligned}
$$

The stability of the controller needs to be analyzed during the switch from 'congested traffic' to 'free flow'. Basically, in case of congestion, the controller remains in controller mode 2 in saturation effect, until density value $\rho_{\text {sat,low }}$ is reached. The controller remains in mode 2 ('congested traffic'), reducing ramp flow until the mainstream density drops below the lower threshold value $\rho_{\text {sat,low. }}$. When switching back to controller mode 1 ('free flow'), the ramp flow is increased so that a state constraint of a stable value is maintained, always lower than the upper threshold value $\rho_{\text {sat,low }}$. The controller is only switched back in mode 2 when an extreme disturbance appears in the main line. The additional traffic of ramp flow in mode 1 cannot lead to densities higher than $\rho_{\text {sat,high }}$. Thus, the adoption of the hysteretic behavior of the system in the choice of the threshold values leads to the exclusion of the oscillation in controller behavior.

\subsection{Controller mode no. 1 - ramp metering for concentration limiting}

\subsubsection{Cost function}

The cost function of the controller handles the operation of the ramp and the resulting ramp queue. Thus it formalizes the objective to allow as much of the ramp demand as possible to the main line so that the concentration constraint is fulfilled while the ramp queue is minimized for each $\ell=1, \ldots, K$ control horizon step. Formally:

$$
J(k)=\sum_{\ell=1}^{K} \sum_{i=1}^{N_{s}}\left(\left\|l_{i}(k+\ell)\right\|_{2}^{2}+\omega_{1}\left\|o_{i}(k+\ell)-r_{i}(k+\ell)\right\|_{2}^{2}+\omega_{2}\left\|r_{i}(k+\ell-1)-r_{i}(k+\ell)\right\|_{2}^{2}\right) .
$$

When tuning weighting parameters $\omega_{1}$ and $\omega_{2}$, Bryson's rule is followed, see Franklin et al. (2002.). For any $\chi_{i}$ term within the cost function, the weight $\omega_{i}=1 / \chi_{i \text {,max }}^{2}$ is chosen, where $\chi_{i, \max }$ is the maximal nominal value of the term $\chi_{i}$. The initial weighting parameters are then manually fine-tuned and normalized by $\max \left\{\omega_{i}\right\}$. (The resulting parameters are $\omega_{1}=1, \omega_{2}=0.1$ ).

\subsubsection{Constraints}

The following constraints are set for the system variables.

- The specification of state constraints is a key element of the design of the first controller mode as they represent the prescribed concentration limits:

$$
c_{j}^{p}(k+\ell) \leq c_{\text {limit }}^{p},
$$

for each $\ell=1, \ldots, K$ sample step and each $j=1, \ldots, \mathcal{N}_{b}$ balance volume. $c_{\text {limit }}^{p}$ is determined by the contribution of the motorway to the local pollution of pollutant $p$, the value is usually a fraction 
of the legislation limit. However, the sensitivity of the concentration dynamics is low for the control inputs $r_{i}$ and $v s l_{i}$ (ramp metering and variable speed limit of segment $i, i=1, \ldots, N_{s}$ ) (see Csikós et al. (2013b)), thus the optimization problem is ill-conditioned for the concentration constraints.

A solution for the problem is to apply state constraints directly on the traffic variables. This can be realized through the boundary concentration values of the emission dispersion model as they can also be considered as external excitations of the dispersion system. By solving the discrete concentration dynamic equation (8) for steady-state conditions, the maximal emission can be expressed for a specified $c_{\text {limit }}^{p}$ concentration constraint as an external excitation:

$$
\varepsilon_{\text {limit }}^{p}(k)=c_{\text {limit }}^{p} H_{j} L_{j} W_{j} \frac{w(k)+\lambda_{j}(k) X_{j}}{w(k)} .
$$

In the following, we assume that the emission of the ramp queue is negligible compared to that of the main lane. Hence, the macroscopic emission distribution $\varepsilon^{p}$ becomes a function of traffic density and mean speed. By substituting (5) to (14) and by further analysis of measurement data, traffic state constraints can be chosen that satisfy (14).

A relationship sought between the emission limit $\varepsilon_{\text {limit }}^{p}$ and the traffic variables. For this end $\varepsilon^{p}(\rho, v)$ is illustrated in Figure 3, assuming the model parameters given in Section 4. Emission values are represented in color scale for the corresponding traffic state (speed and density) pairs. Also, measured values of the traffic states are highlighted with dots.

The primary state variable of the traffic system is the traffic density, with the speed as a secondary variable, expressing its dynamics around the equilibrium speed function. Below the critical density (i.e. free flow conditions), the static dependence of traffic mean speed on traffic density is present in a dominant way. As a conclusion, below the critical density - which is the operation domain of this controller mode - traffic speed, and also, macroscopic emission (which is a function of traffic density and speed) can be accurately approximated as a function of the traffic density variable only. Hence, the maximal macroscopic emission can also be represented as a function of the traffic density, thus the state constraints should be specified for the density values. The traffic density constraint is thus stated so that the highest emission level is a

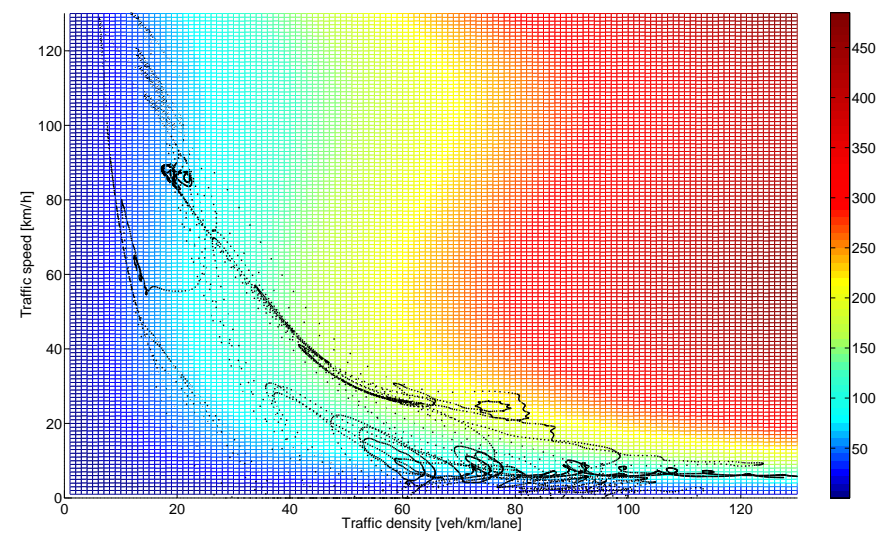

Figure 3: Macroscopic emission function and measurements of flow variables

function of traffic density. For this function, the envelope of the highest emission values for pollutant $p$ can be considered based on substituting traffic measurement data to the emission function (5):

$$
\varepsilon_{\text {max }}^{p}=\max _{\forall v_{i}} \varepsilon_{i}^{p}\left(\rho_{i}, v_{i}\right) .
$$

For each pollutant $p$, the maximal traffic density can be obtained as follows:

$$
\rho_{i}^{p}=\arg \left(\varepsilon_{\max }^{p}(\rho)\right) .
$$


The ultimate density constraint for segment $i$ is the lowest of the density bounds of $\rho_{i}^{p}$ :

$$
\rho_{i}(k+\ell) \leq \min _{p} \rho_{i}^{p}(k),
$$

for each $k$ sample step and each $\ell=1, \ldots, K$ control horizon step.

- Throughout the control horizon, constant disturbance values are considered. Thus,

$$
\begin{aligned}
q_{0}(k+\ell) & =q_{0}(k), \\
v_{0}(k+\ell) & =v_{0}(k), \\
\rho_{N_{i}+1}(k+\ell) & =\rho_{N_{i}+1}(k), \\
o_{i}(k+\ell) & =o_{i}(k), i=1, \ldots, N_{s}, \\
s_{i}(k+\ell) & =s_{i}(k), i=1, \ldots, N_{s}, \\
w(k+\ell) & =w(k) .
\end{aligned}
$$

for each $k$ sample step and each $\ell=1, \ldots, K$ control horizon step.

- Regarding the inputs, ramp metering is constrained between zero and the possible admissible traffic flow:

$$
0 \leq r_{i}(k+\ell) \leq \min \left(S, o_{i}(k)+\frac{l_{i}}{T}\right),
$$

for each $k$ sample step and each $\ell=1, \ldots, K$ control horizon step. $S$ denotes the saturation flow $(S=1800 \mathrm{veh} / \mathrm{h})$, the highest possible flow of a starting traffic. The formula $o_{i}(k)+\frac{l_{i}}{T}$ gives the highest admissible flow from queue $i$ in units [veh/h]. In this mode the VSL control inputs are fixed to:

$$
\operatorname{vsl}_{i}(k+\ell)=130, i=1, \ldots, N_{s}, \ell=1, \ldots, K \text {. }
$$

\subsection{Controller mode no. 2 - traffic stabilization}

\subsubsection{Cost function}

For the cost function, a similar approach is used as in Csikós et al. (2013a). The main goal is the stabilization of the mainstream states with the neglect of ramp queue lengths (however, implicitly, by minimizing the difference between the ramp demands and actual ramp flows the queue length is minimized). By this, a regulation control is realized for the setpoint $\rho_{c r}$. The last two terms of the cost function are responsible for the suppressing of spatial and temporal oscillation in VSL inputs. The cost function is given by the following functional:

$$
\begin{aligned}
J(k)= & \sum_{\ell=1}^{K}\left(\sum_{i=1}^{N_{s}}\left\|\rho_{i}(k+\ell)-\rho_{\text {crit }}\right\|_{2}^{2}\right. \\
& +w_{1} \sum_{i=1}^{N_{s}}\left\|o_{i}(k+\ell)-r_{i}(k+\ell)\right\|_{2}^{2} \\
& +w_{2} \sum_{i=1}^{N_{s}}\left\|v l_{i}(k+\ell)-v s l_{i}(k+\ell-1)\right\|_{2}^{2} \\
& \left.+w_{3} \sum_{i=1}^{N_{s}-1}\left\|v s l_{i}(k+\ell)-v s l_{i+1}(k+\ell)\right\|_{2}^{2}\right) .
\end{aligned}
$$

The values of the tuned weighting parameters are as follows: $w_{1}=0.02, w_{2}=0.02, w_{3}=0.05$.

\subsubsection{Constraints}

- No constraints are set on the states.

- Similarly to controller mode 1 , throughout the control horizon, constant disturbance values are considered (see (16)).

- Inputs

For the ramp control: the same conditions are used as in controller mode no. 1, see eq. (17). VSL inputs may take values from the following discrete set:

$$
\text { vsl }_{\text {set }}=\{60,70,80,90,100,110,120,130\} .
$$


Remark 2 The design of the optimal VSL input is carried out in a continuous manner. Then, the applied control is chosen by rounding the designed input to the possible discrete values. For the elimination of VSL input oscillation a two-step optimization is carried out. In the first step, optimal input is calculated, considering a continuous set for VSL input. Then, input variables for VSL control are rounded to the elements of the discrete set. After setting the discrete values, another optimization is run solely for the ramp control, considering the fixed VSL values as input constraints.

\section{Case study}

In this section the behaviour of the proposed controller is investigated. For the sake of a concise study, a complex scenario is simulated with changing demands and constraints. The following aspects are examined: i) the performance and effectiveness of the control modes under changing demands and constraints; ii) the switching performance of the hybrid controller in terms of response time, mode choice and stability.

\subsection{Simulation setup}

The case study presents a traffic situation with unstable conditions: a rush hour scenario during which two shockwaves of different amplitudes appear. Furthermore, a drop in upstream main lane load is featured for a short period of time, which gives an opportunity to show that the controller can compensate the traffic load from the queued vehicles of the ramp.

The simulation network is a $10 \mathrm{~km}$ long, two-lane motorway stretch, divided to equal segment lengths. The network is controlled with a ramp at the first segment (see Fig. 4) and variable speed limits at each segment.

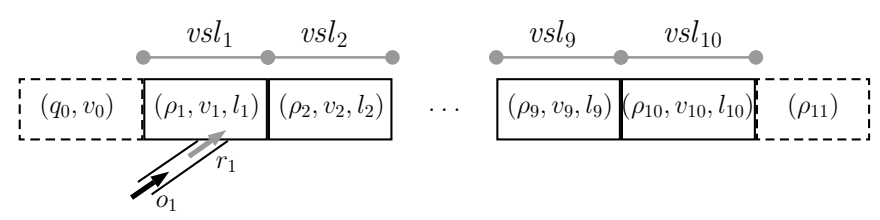

Figure 4: Case study network layout

For simplicity, wind direction is perpendicular to the motorway, and the parameters of the flow channels are equally $X_{j}=1000 \mathrm{~m} ; H_{j}=30 \mathrm{~m} ; L_{j}=1000 \mathrm{~m}$. This simplified topology gives a platform to a thorough evaluation of the proposed controller. The parameters of the traffic model used for the simulations are as follows: $a=2.5, \rho_{c r}=25 \mathrm{veh} / \mathrm{km} / \mathrm{lane}, \tau=0.005, \eta=65$, $\delta=1.68, \kappa=40$.

The disturbances of the traffic system during the simulation are plotted in Fig. 5. A constant $w=4 \mathrm{~m} / \mathrm{s}$ for wind speed and $s_{i}=0, i=1, \ldots, N_{s}$ off-ramp flow values are used. 

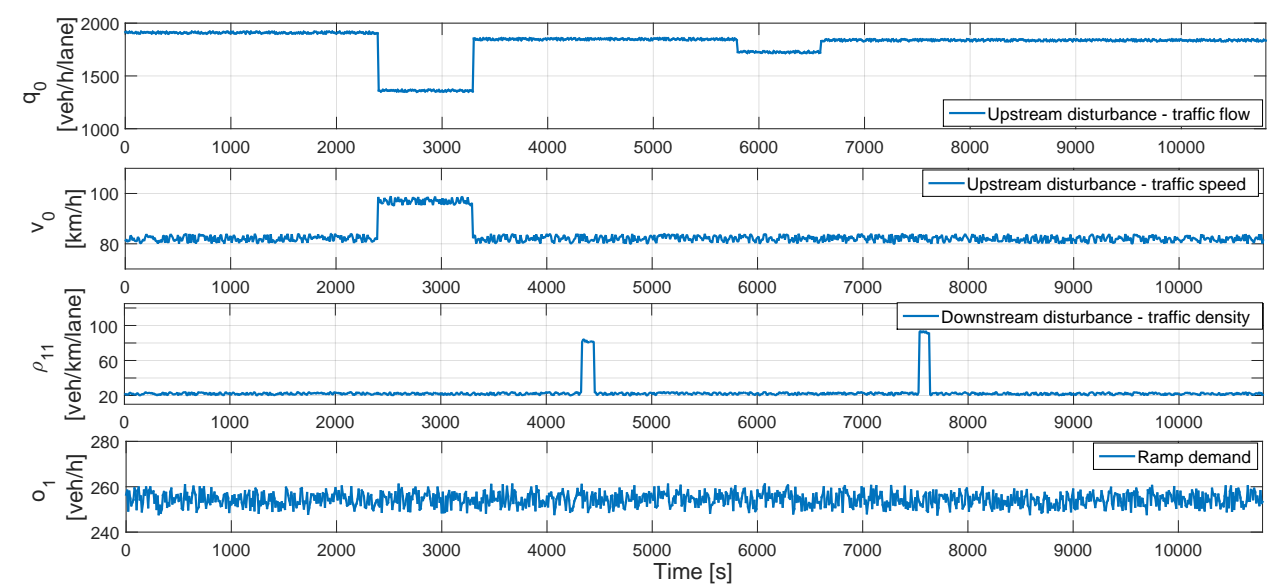

Figure 5: Disturbances: upstream flow and mean speed; downstream traffic density; on-ramp demand

The emission factor function of $\mathrm{CO}$ pollution (representing the average fleet of Hungary) is used in the form: ef ${ }^{C O}(t)=0.00334 v(t)^{2}-0.8 v(t)+58$. The function is determined based on the average fleet composition of Hungary, applied for the Copert average-speed emission model Ntziachristos et al. (2000). For the idling emission rates, the following value is used: $e^{\mathrm{CO}}=7.123$ $\mathrm{g} / \mathrm{h} / \mathrm{veh}$ (determined for the Hungarian fleet composition using Office of Transportation and Air Quality (2008)).

The specification of the concentration limits is a delicate matter, as motorway traffic cannot be considered the sole source of air pollution. In our approach, we follow the figures of Commission (2016). Road transport was responsible for $31.7 \%$ of the air pollution of the EU in 2012. Considering that $50 \%$ of traffic emissions come from motorway networks, the contribution of this road type can be estimated as $15.85 \%$ to overall pollution. The legislation limit of CO for 1-hour daytime period is $0.035 \mathrm{~g} / \mathrm{m}^{3}$, and the fraction that motorway traffic can reach is chosen proportionally as $0.0055 \mathrm{~g} / \mathrm{m}^{3}$. The concentration limits are prescribed as follows:

$$
c_{\text {limit }}^{\mathrm{CO}}= \begin{cases}0.0095 \mathrm{~g} / \mathrm{m}^{3}, & \text { if } t<1200 \mathrm{~s} \\ 0.0055 \mathrm{~g} / \mathrm{m}^{3}, & \text { if } t \geq 1200 \mathrm{~s}\end{cases}
$$

The changing limits provide an opportunity to analyze the transients of the controller during state constraint changes.

For the controller, the decision values of eq. (12) are obtained by manual tuning following a set of test runs for the case study system. As a result of the tuning, the choice of $\rho_{\text {sat,low }}=23$ $\mathrm{veh} / \mathrm{km} /$ lane and $\rho_{\text {sat }, \text { high }}=28 \mathrm{veh} / \mathrm{km} /$ lane is made.

\subsection{Simulation results}

The traffic density and CO concentration profiles of the uncontrolled scenario are depicted in Figs. 6 and 7. The effect of the disturbances can be observed: the drop of upstream main lane load between 2400-3300 s results in a lower traffic density and also lower concentration values. The peaks in the downstraeam disturbance at $4300 \mathrm{~s}$ and $7600 \mathrm{~s}$ result in heavy shockwaves. 


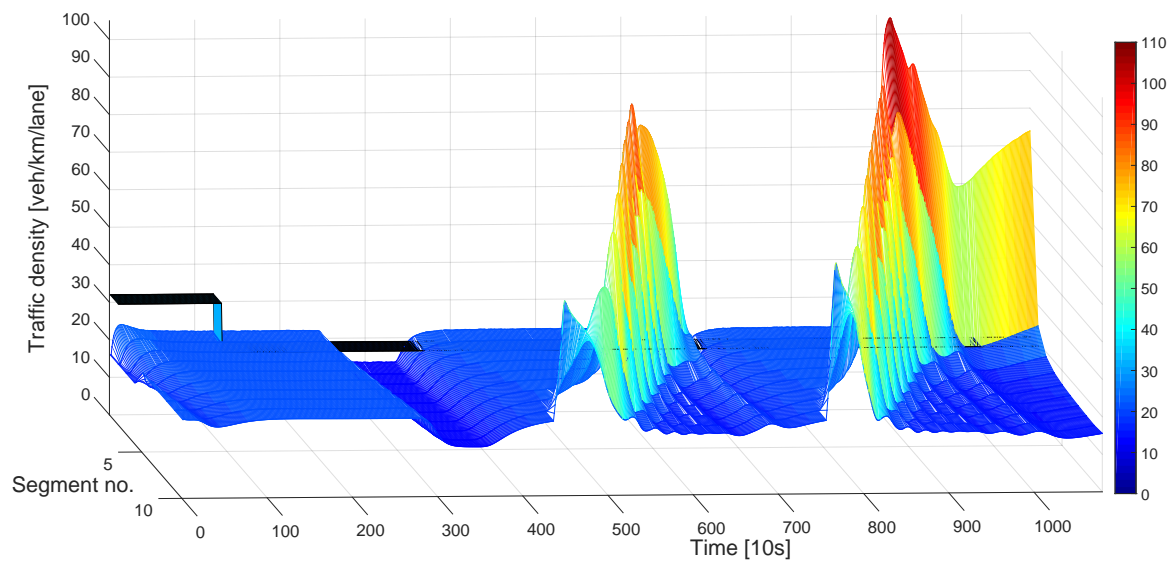

Figure 6: Traffic density - uncontrolled case

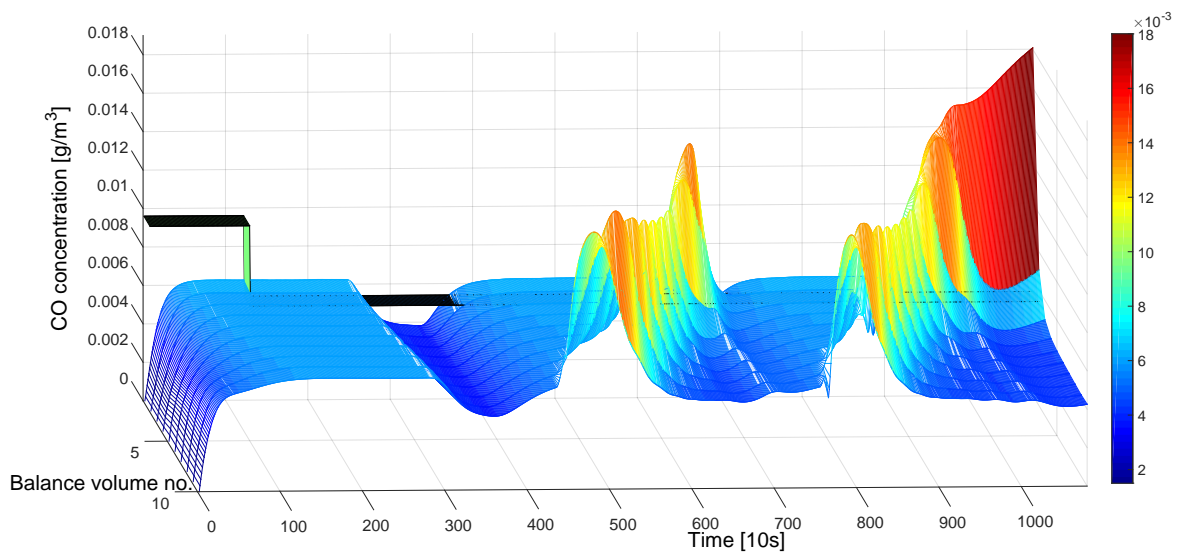

Figure 7: CO concentration limits and values - uncontrolled case

The controller analysis is presented in two scenarios:

- In the first scenario, controller mode no. 1 of the hybrid controller is used only.

- In the second scenario both modes of the hybrid controller are applied, switched by the finite automata.

The motivation for the comparison is to highlight the differences between controller setups during changing conditions. It is expected that although controller mode no. 1 can handle changing traffic loads to an extent, however, by itself it is not able to eliminate both shockwaves, while the hybrid controller can maintain limited concentrations and is capable of stabilizing traffic by deploying both of its control modes. 


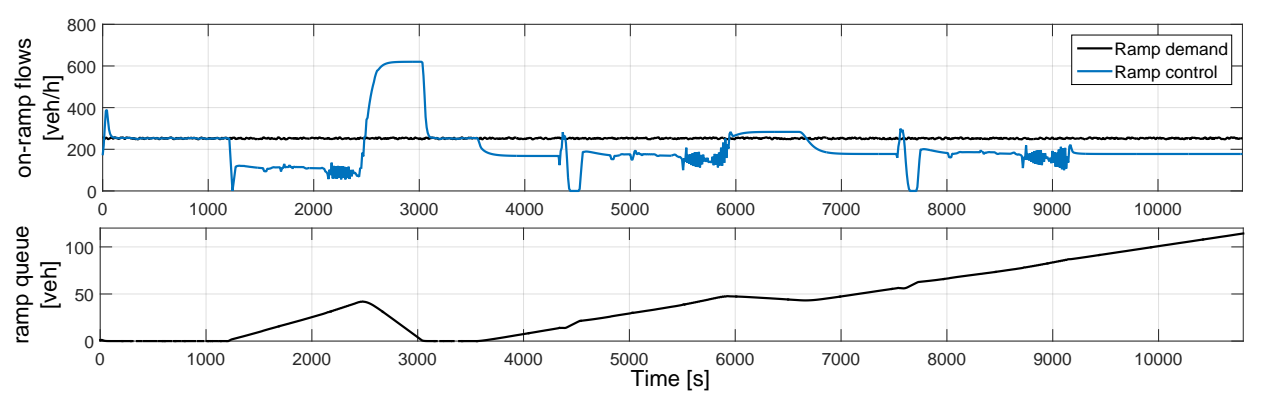

Figure 8: Ramp control and ramp queue - controller mode 1

First control scenario - using only controller mode 1 In case of using the mode no. 1 only, two main observations can be taken: first, the controller is capable of satisfying its control objective with a good performance; and second, although, it is able to adapt to certain changes in state constraints and disturbances under stable conditions, it cannot stabilize traffic in case of extreme disturbances.

During 0-4300 s and 5000-7600 s, traffic is under free flow conditions, and the pollutant concentrations remain below the pre-specified limits. The restriction in limits is effectively addressed at $1200 \mathrm{~s}$, see Fig. 8. However, at this point, concentration values show a longer settling time than traffic density due to the longer time constant of the dispersion dynamics, see Fig. 9 and 10. During 2500-3000 s, upstream demand of the main lane is decreased, thus overall flow is complemented by an increased ramp flow, supplied by the ramp queue.

A considerable relief is experienced in the first shockwave at $4300 \mathrm{~s}$, however, the controller is not capable of suppressing the second one. During the shockwaves the extreme densities lead to a violation of concentration limits. In such cases, the positive input constraints are superior to the state constraints.

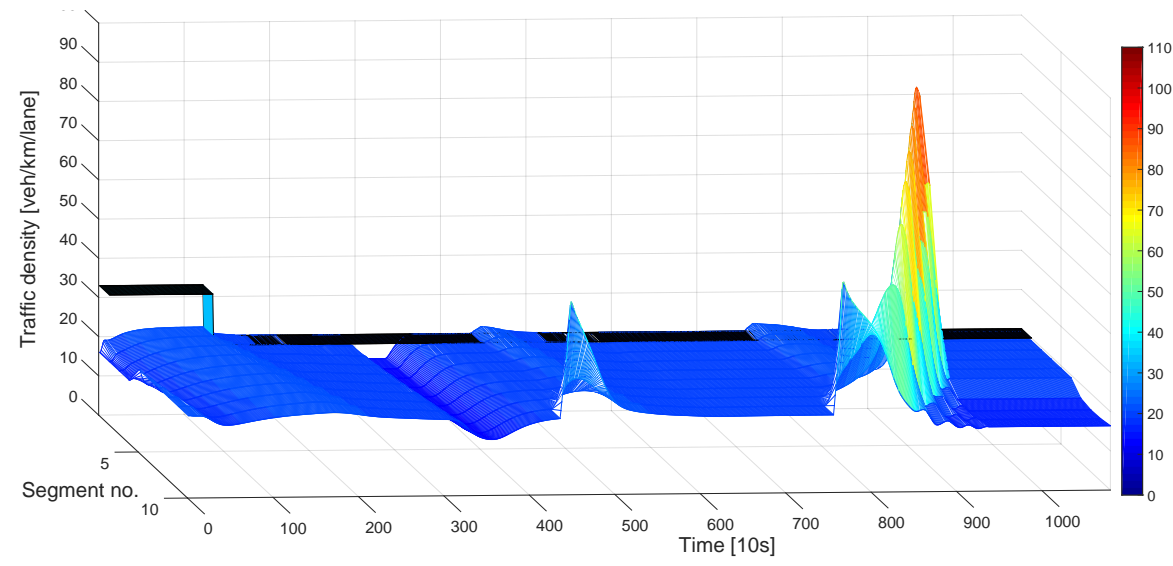

Figure 9: Traffic density and prescribed limits - controller mode 1 


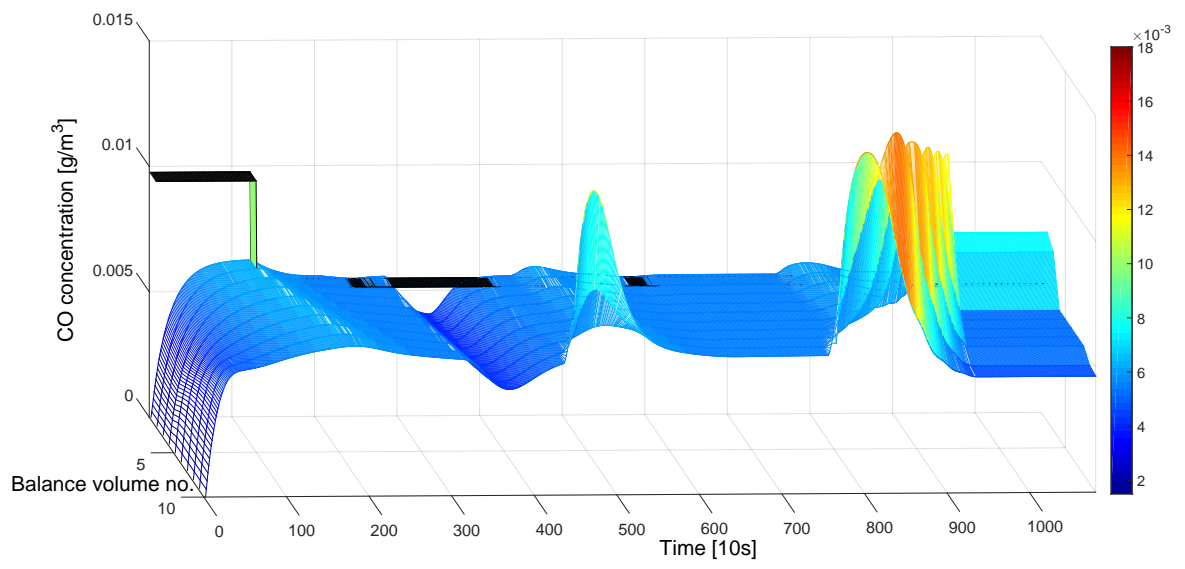

Figure 10: Concentrations and prescribed limits - controller mode 1

Second control scenario - hybrid controller When using the hybrid controller, both controller modes are applied, switched by the finite automata. The appropriate switching of the control modes leads to an effective handling of changing conditions. During the scenario, mainly control mode 1 is in operation (see Fig. 12), switching to mode 2 during shockwaves at $4300 \mathrm{~s}$ and $7700 \mathrm{~s}$ only. In both instances, the switched operation results in a fast response and opposite to control mode 1 only, an effective stabilization of the flow can be observed by using ramp metering and VSL control (see Figs. 11, 13 and 14).

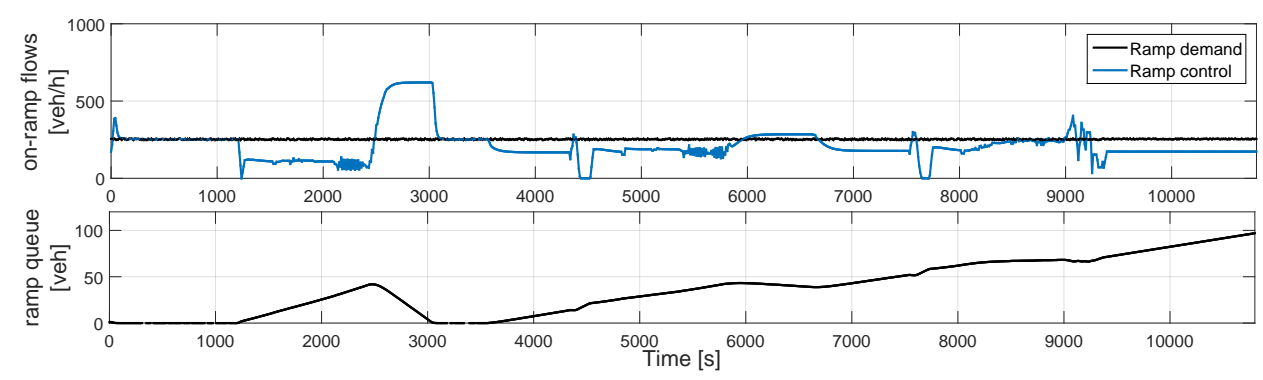

Figure 11: Ramp input and queue of the hybrid controller
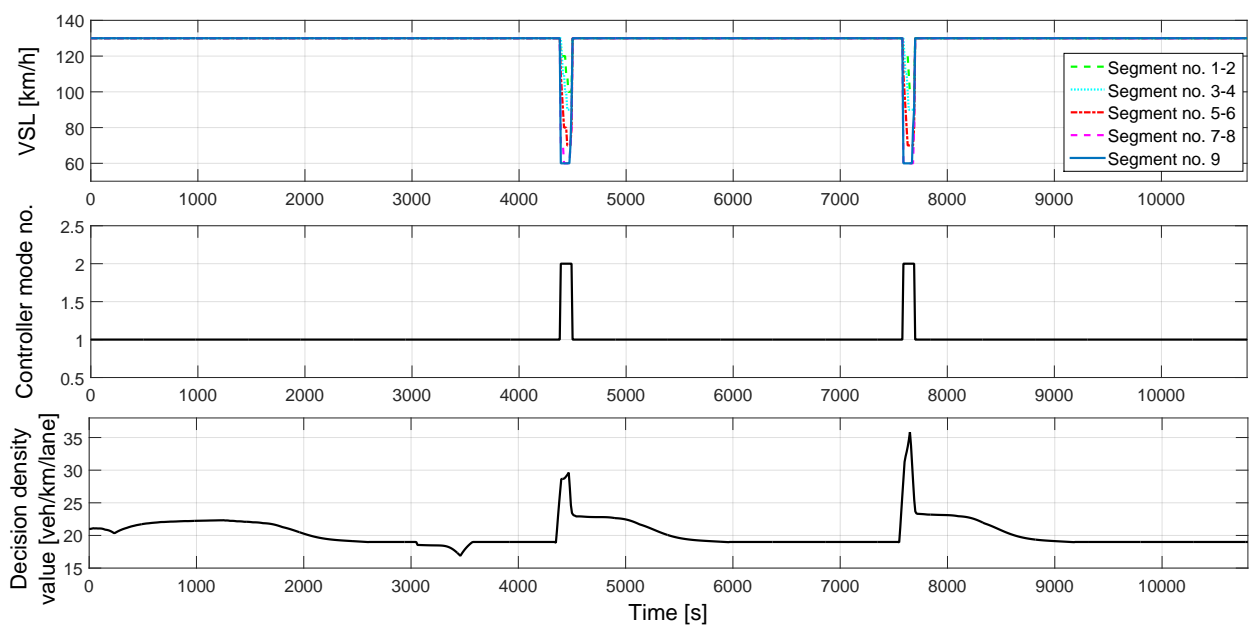

Figure 12: VSL inputs, decision density value and switching mode no. - two-mode control 


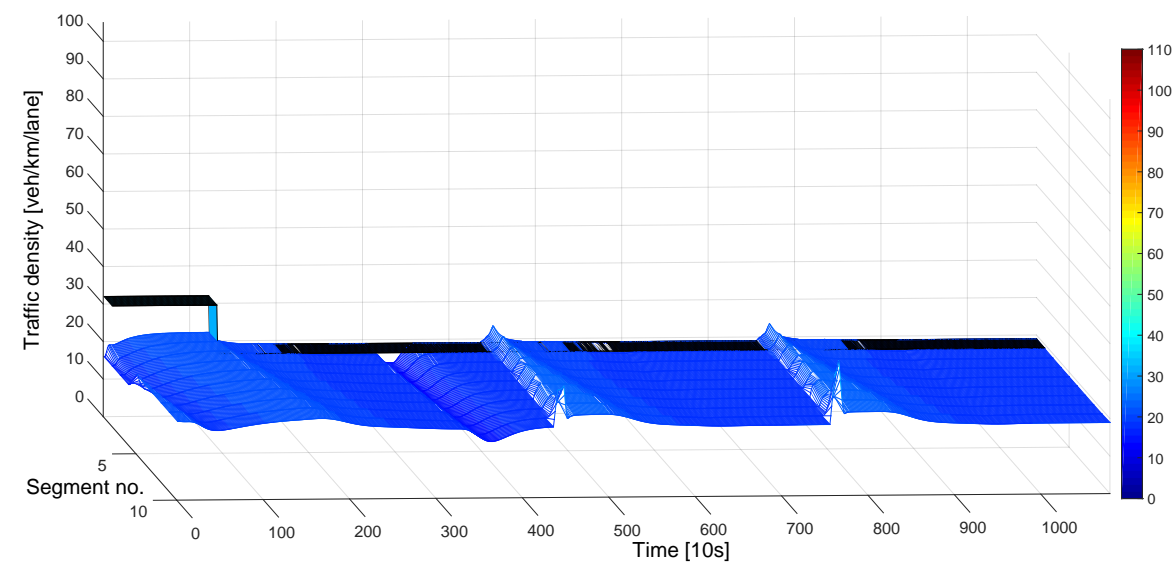

Figure 13: Traffic density and prescribed limits - two-mode control

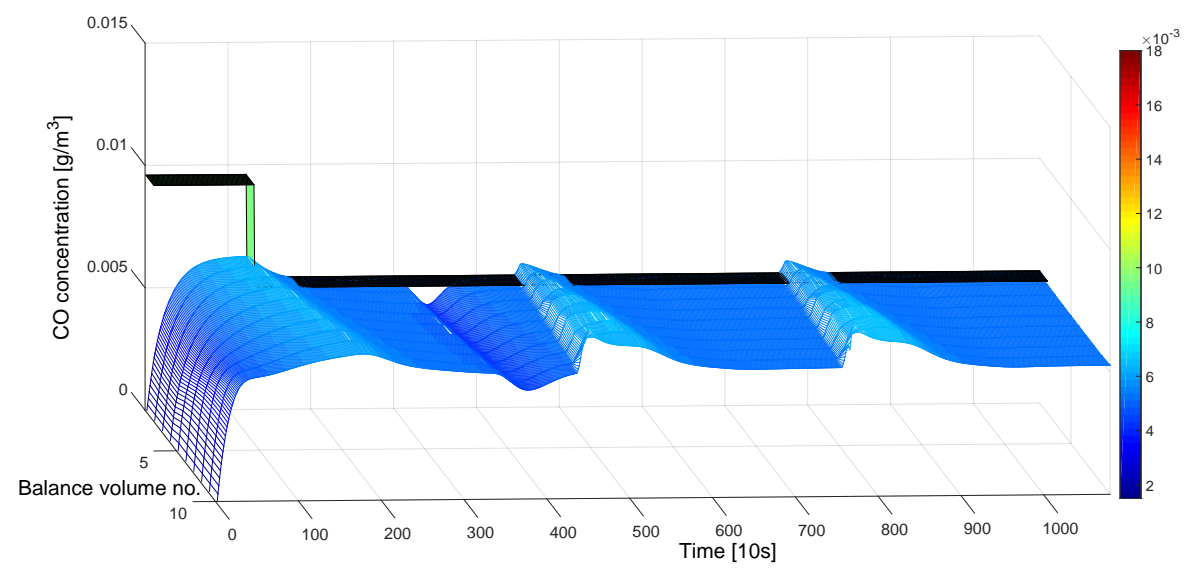

Figure 14: Concentrations and prescribed limits - two-mode control

The switching dynamics, alongside the ramp and VSL input signals for both switches are magnified in Fig. 15. During the shockwaves, operation mode no. 2 is in effect for $120 \mathrm{~s} / 220 \mathrm{~s}$ respectively, with moderate speed limits. The speed limit signals show appropriate the spatial and temporal gradients, and no oscillation is present. 


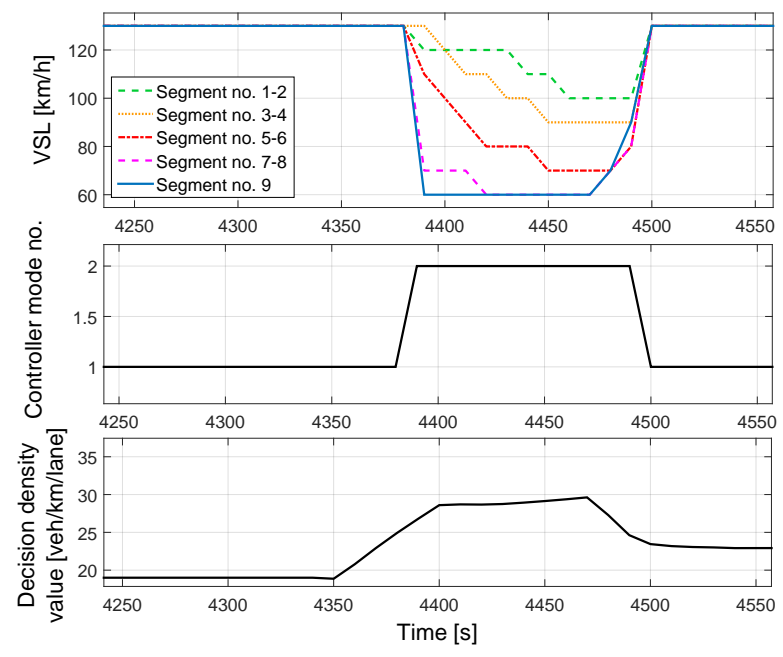

(a) First shockwave

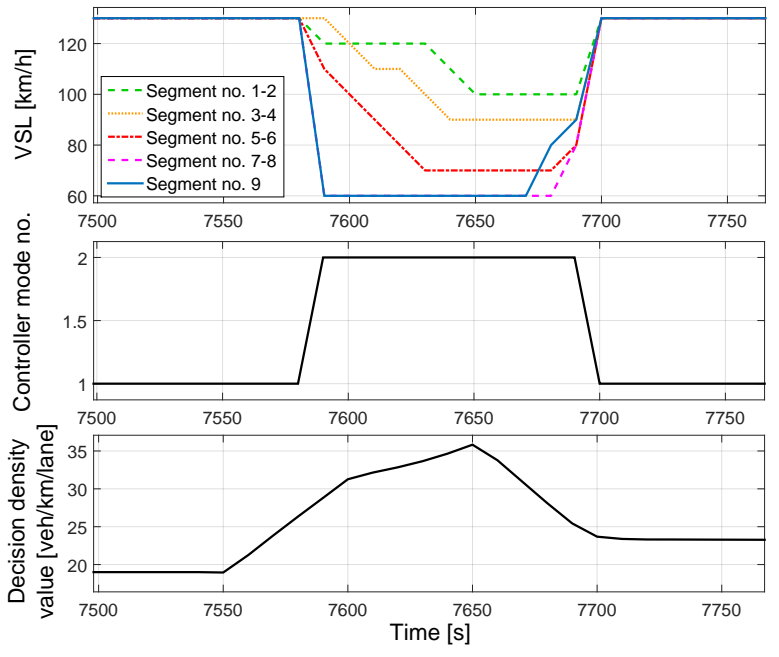

(b) Second shockwave

Figure 15: Operation of VSL control during shockwaves

In Fig. 16 the total time spent (TTS) of the considered network, involving on-ramps is depicted for the three analyzed scenarios. The highest peaks of TTS can be observed in the uncontrolled case, whereas both controlled cases reduce real-time TTS by appropriately homogenizing flow conditions along the motorway. The effect of smaller shockwaves (with an amplitude of the first one) can be suppressed by operation mode no. 1, using ramp metering only. However, this mode cannot handle the second shockwave, and as a result a considerable increase in TTS can be observed, due to the closing of on ramps and the unsolved traffic jam (during 7500-9200s). The hybrid approach however exploits the effective homogenization effect of speed limit control and stabilizes the network with low TTS values. The aggregated TTS values of the uncontrolled and controlled cases are summarized in Table 1.

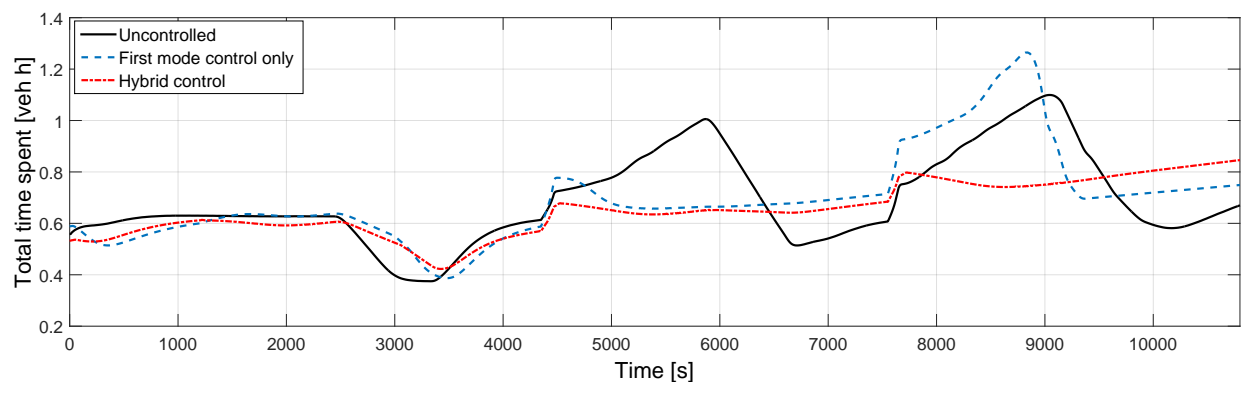

Figure 16: Total time spent in network

\begin{tabular}{lccc}
\hline - & Uncontrolled & Control mode 1 only & Hybrid control \\
\hline TTS [veh h] & 745.98 & 750.63 & 704.78 \\
\hline
\end{tabular}

Table 1: Total time spent in network over simulation

\section{Computational analysis of the control system}


The computational complexity of the control modes is analyzed through their underlying optimization problems. The optimization parameters are summarized in Table 2. Both problems are NP-hard due to the nonlinear equality constraints, however, two important differences can be pointed out. First, controller mode 1 has a higher number of constraint variables as its state vector is extended by the dispersion dynamics. This difference by itself means a polynomial increase in computation time in favor of controller mode 2. Second, control mode 2 features a two-step optimization. The first optimization step of controller mode 2 has a higher number of decision variables than the problem of mode 1 , leading to an exponential increase in computation time for a fixed control horizon $K$. As a result of the above reasons, control mode 2 has a significantly higher computational demand than that of controller mode 1.

\begin{tabular}{lccc}
\hline & Control mode 1 & \multicolumn{2}{c}{ Control mode 2 } \\
\cline { 3 - 4 } & & Optim. step 1 & Optim. step 2 \\
\hline Number of constraints & $2 N_{s}+N_{r}+3 \mathcal{N}_{b}$ & $2 N_{s}+N_{r}$ & $2 N_{s}+N_{r}+N_{v}$ \\
Horizon length & $K$ & $K$ & $K$ \\
Number of optimized inputs & $N_{r}$ & $N_{r}+N_{v}$ & $N_{r}$ \\
Number of decision variables & $K N_{r}$ & $K\left(N_{r}+N_{v}\right)$ & $K N_{r}$ \\
\hline
\end{tabular}

Table 2: Parameters of the optimization problems

In the case study, the optimization of the nonlinear model predictive control is carried out by the active set algorithm (via fmincon in MatLab, as suggested in Grune \& Pannek (2011)). For prediction horizon length, the value $K=5$ is chosen. The parameters of the optimization problems and the corresponding computational times for the case study of Section 4 are summarized in Table $3^{2}$.

\begin{tabular}{lccc}
\hline & Control mode 1 & \multicolumn{2}{c}{ Control mode 2 } \\
\cline { 3 - 4 } & & Optim. step 1 & Optim. step 2 \\
\hline Number of constraint variables & 51 & 5 & 26 \\
Horizon length & 5 & 6 & 5 \\
Number of optimized inputs & 1 & 30 & 5 \\
Number of decision variables & 5 & \multicolumn{2}{c}{2.342} \\
\cline { 3 - 4 } Computation time of a sample step $[\mathrm{s}]$ & 0.468 & \multicolumn{2}{c}{5} \\
\hline
\end{tabular}

Table 3: Computational parameters of the case study

Table 4 summarizes the aggregated results of different horizon lengths, for values $K=1, \ldots, 5$. Further increase in the horizon length did not lead to considerable performance improvement, but a significant increase of CPU times. In addition to TTS, the following performance metrics is used for analysis: $P_{v}$ denotes the percentage over the spatiotemporal domain of analysis during which the concentration limits are violated. The increase of $K$ leads to a slight improvement in TTS, whereas a more significant reduction in $P_{v}$. The reason for this is the higher time constant of the dispersion dynamics, which reacts with long transients to the change in excitation (see e.g. Fig. 10). By increasing $K$, a higher weighting is put on concentration tracking error, and the violation of concentration limits can be reduced.

Regarding the convexity of the problem, an analysis was carried out to find out the existence of a globally optimal solution. According to several runs from different initial solutions, the

\footnotetext{
${ }^{2}$ Simulations are performed on a personal computer with Intel Core i5 3.0-GHz CPU and 8 GB of RAM.
} 


\begin{tabular}{cccc}
\hline$K$ & Avg. CPU time/step [s] & TTS [veh h] & $P_{v}[\%]$ \\
\hline 1 & 0.1147 & 741.01 & 23.4 \\
2 & 0.2519 & 737.26 & 25.6 \\
3 & 0.3056 & 733.21 & 22.3 \\
4 & 0.4167 & 717.85 & 17.4 \\
5 & 0.5273 & 704.78 & 16.2 \\
\hline
\end{tabular}

Table 4: Comparison of prediction horizons

same solution was obtained. However, by itself this does not prove that the stated optimization problem is convex.

\section{Conclusion}

In this work a control system is developed and analyzed for the suppression of motorway shockwaves and the reduction of pollutant concentrations of inhabited areas near motorways. The dynamic model of the controlled system is based on the second-order macroscopic model description of the freeway traffic, joined by the emission dispersion model introduced in Csikós et al. (2015).

Based on a preliminary analysis of control objectives and control measures, different controller setups were used for the considered traffic situations. During free flow, when stable conditions are present concentration limiting can be aimed. This objective can be achieved by ramp metering control, whereas no dynamic speed limit is used, since it influences emission distribution in an undesired way. In case of congestion along a moving jamwave, variable speed limit control is also needed in addition to ramp metering. In this case the objective is flow stabilization, and concentration limiting is not considered. These significantly different controller setups call for the use of a hybrid controller. For the above tasks dedicated control modes were developed, between which a finite automata is responsible for switching.

The behaviour of the proposed hybrid control system is analyzed in a complex case study, from which the following conclusions can be drawn. The controller setup for the different objectives is proven to be appropriate by the performance of the control modes. However, their capabilities are restricted to certain state domains. The hybrid controller, by an appropriate switching of the control modes, can adapt to changing demands as well in both the stable and unstable state domain. Furthermore, the finite automata, tuned with consideration of the hysteresis in the system dynamics leads to a stable operation of the controller, i.e., no oscillation in switching emerges. Further analysis shows that the presented model and control system offers a computationally effective centralized control solution which is a result of the simple dynamic model for dispersion.

\section{Appendix}

\section{A Derivation of dispersion dynamics}

The outlined process system can be modeled through the conservation of pollutant masses within the balance volumes. Each flow channel is considered as an autonomous balance volume with individual dynamics. In the following, the conservation is formalized for an arbitrary flow channel $j$. The mass balance for pollutant $p$ in flow channel $j$ is described through the variable $m_{j}^{p}(x, t)$ (measured in units $\left.[\mathrm{g}]\right)$ as a bivariate function of time $(0 \leq t)$ and space $\left(0 \leq x_{j} \leq X_{j}\right)$, where $X_{j}$ denotes the length of flow channel $j$. 
The conservation equation of pollutant $p$ for balance volume $j$ is:

$$
\frac{\partial m_{j}^{p}\left(x_{j}, t\right)}{\partial t}=\phi_{j, \text { in }}^{p}\left(x_{j}, t\right)-\phi_{j, \text { out }}^{p}\left(x_{j}, t\right)-\psi_{j, \text { dis }}\left(x_{j}, t\right)
$$

where $\phi_{j, \text { in }}^{p}\left(x_{j}, t\right), \phi_{j, \text { out }}^{p}\left(x_{j}, t\right)$ and $\psi_{j, \text { dis }}\left(x_{j}, t\right)$ are the inflow, outflow and dissolution rate of the pollutant, respectively.

The inflow of pollutants at the border of the balance volume comes from the emission of the segments involved in the balance volumes:

$$
\phi_{j, i n}^{p}(0, t)=\sum_{i=1}^{N_{j, i}} \varepsilon_{j, i}^{p}(t) \cdot L_{j, i}
$$

where $N_{j, i}$ denotes the number of segments involved in balance volume $j ; \varepsilon_{j, i}^{p}$, given in units $[\mathrm{g} / \mathrm{km} \cdot \mathrm{h}]$ denotes the spatiotemporal emission of motorway segment $j, i, L_{j, i}$ denotes the length of segment $j, i\left(\sum_{i=1}^{N_{j, i}} L_{j, i}=L_{j}\right)$.

The outflow of pollutants is the direct effect of the wind:

$$
\phi_{j, \text { out }}^{p}\left(x_{j}, t\right)=w \frac{\partial m_{j}^{p}\left(x_{j}, t\right)}{\partial x_{j}}
$$

where $w$ (in units $[\mathrm{m} / \mathrm{s}]$ ) denotes the wind speed.

The dissolution of pollutants is described by the following formula:

$$
\psi_{j, d i s}^{p}\left(x_{j}, t\right)=\lambda\left(x_{j}, t\right) m_{j}^{p}\left(x_{j}, t\right)
$$

where $\lambda_{p}\left(x_{j}, t\right)$ (in units $\left[\mathrm{s}^{-1}\right]$ ) denotes the decay rate of the pollutant $p$. The dissolution of gases is modeled by a simplified version of the Gaussian plume model, see in Luhar \& Patil (1989): pollution decay rate $\lambda\left(x_{j}, t\right)$ is modeled as a function of wind speed and downwind distance, and is obtained from the following formula:

$$
\lambda\left(x_{j}, t\right)=\frac{w(t)}{X_{j} a(w(t)) X_{j}^{b(w(t))} 2 \pi} \int_{-\infty}^{-H_{j} / 2} \exp \frac{-z^{2}}{2 a(w(t)) X_{j}^{2 b(w(t))}} d z
$$

where $X_{j}$ and $H_{j}$ are geometric parameters of flow channel $j$ (see Fig. 1b), $a(w)$ and $b(w)$ are modeling parameters, functions of the wind speed, see Schwede (1999).

The relationship between the mass of pollutant $p$ and its concentration in an infinitesimal segment $\Delta V_{j}$ of balance volume $j$ is described as:

$$
\Delta m_{j}^{p}\left(x_{j}+\Delta x_{j}, t\right)=c_{j}^{p}\left(x_{j}+\Delta x_{j}, t\right) \cdot \Delta V_{j}=c_{j}^{p}\left(x_{j}+\Delta x_{j}, t\right) \cdot H_{j} L_{j} \Delta x_{j}
$$

where $c_{j}^{p}\left(x_{j}+\Delta x_{j}, t\right)$ denotes the concentration of pollutant $p$ in the balance volume increment $\Delta V_{j}$, measured in units $\left[\mathrm{kg} / \mathrm{m}^{3}\right]$.

By using eqs. (21)-(25), (20) can be reformalized in one continuous partial differential equation taking the limit $\Delta x_{j} \rightarrow 0$ :

$$
\frac{\partial c_{j}^{p}\left(x_{j}, t\right)}{\partial t}=-w(t) \frac{\partial c_{j}^{p}\left(x_{j}, t\right)}{\partial x_{j}}-\lambda_{p}\left(x_{j}, t\right) c_{j}^{p}\left(x_{j}, t\right)
$$

Initial condition of the partial differential equation is supposed as follows:

$$
c_{j}^{p}\left(x_{j}, 0\right)=0, \forall x_{j} \in\left[0, X_{j}\right]
$$


The boundary condition of eq. (26) is obtained from the concentration emerging at the boundary of the balance volume, derived from the absorption in the volume $H_{j} \times L_{j} \times W_{j}$ :

$$
-\left.w(t) \frac{\partial c_{j}^{p}\left(x_{j}, t\right)}{\partial x_{j}}\right|_{x_{j}=0}=\frac{\sum_{j, i=1}^{N_{j, i}} \varepsilon_{j, i}^{p}(t) \cdot L_{j, i}}{H_{j} L_{j} W_{j}}
$$

The continuous partial differential equation (26) is then converted into a set of ordinary difference equations through the spatial and temporal discretization. Here, attention needs to be payed to the numeric stability of the solution, by applying the Courant-Friedrichs-Lewy condition (see Courant et al. (1928)) to the sampling.

The discrete dynamics of concentration in balance volume $j$ for time step $k$ is stated as:

$$
c_{j}^{p}(k+1)=c_{j}^{p}(k)+T\left(w(k) \frac{c_{j}^{p, 0}(k)-c_{j}^{p}(k)}{X_{j}}-\lambda_{j}(k) c_{j}^{p}(k)\right)
$$

The discrete state dynamics are accompanied with the conditions at the boundary and the initial conditions. The initial condition is given by:

$$
c_{j}^{p}(0)=0, \forall x_{j} \in\left[0, X_{j}\right]
$$

Boundary condition $c_{j}^{p, 0}(k)$ is stated based on the macroscopic emissions emerging at the root of the balance volume as in eq. (27) in discrete form:

$$
c_{j}^{p, 0}(k)=T \frac{\sum_{j, i=1}^{N_{j, i}} \varepsilon_{j, i}^{p}(k) L_{j, i}}{H_{j} L_{j} W_{j}}
$$

\section{Acknowledgements}

This project is supported by the National Research, Development and Innovation Office (NKFIH) through grant No. 115694 which is gratefully acknowledged.

\section{References}

Alsabaan, M., Naik, K. \& Khalifa, T. (2013), 'Optimization of fuel cost and emissions using v2v communications', IEEE Transactions on Intelligent Transportation Systems 14(3), 14491459.

Carolien, B., Panis, L. I., Rudi, T., Davy, J. \& Steven, B. (2007), 'The application of the simulation software vetess to evaluate the environmental impact of traffic measures', 10th Conference on Computers on Urban Planning and Urban Management .

Chamberlin, R., Swanson, B., Talbot, E., Dumont, J. \& Pesci, S. (2011), Analysis of moves and cmem for evaluating the emission impacts of an intersection control change, in 'TRB 90th Annual Meeting', number 11-0673.

Chang, X., Chen, B. Y., Li, Q., Cui, X., Tang, L. \& Liu, C. (2013), 'Estimating real-time traffic carbon dioxide emissions based on intelligent transportation system technologies', IEEE Transactions on Intelligent Transportation Systems 14(1), 469-479.

Chen, Z., Yang, C. \& Chen, A. (2014), 'Estimating fuel consumption and emissions based on reconstructed vehicle trajectories', Journal of Advanced Transportation 48(6), 627-641.

Coensel, B. D., Can, A., Degraeuwe, B., Vlieger, I. D. \& Botteldooren, D. (2012), 'Effects of traffic signal coordination on noise and air pollutant emissions', Environmental Modelling and Software 35, 74-83. 
Commission, E. (2016), E. u. transport in figures: Statistical pocketbook, Technical report, Eurostat.

URL: https://ec.europa.eu/transport/sites/transport/files/pocketbook2016.pdf

Courant, R., Friedrichs, K. \& Lewy, H. (1928), 'Uber die partiellen differenzengleichungen der mathematischen physik', Mathematische Annalen 100(1), 32-74.

Csikós, A. \& Varga, I. (2012), 'Real-time modeling and control objective analysis of motorway emissions', Procedia - Social and Behavioral Sciences 54, 1027-1036.

Csikós, A., Varga, I. \& Hangos, K. M. (2013a), Freeway shockwave control using ramp metering and variable speed limits, in 'Proceedings of the 21st Mediterranean Conference on Control and Automation', Platanias-Chania, Greece. June pp. 1569-1574, pp. 25-28.

Csikós, A., Varga, I. \& Hangos, K. M. (2013b), A simple dynamic model for the dispersion of motorway traffic emission, in 'Proceedings of the 16th International IEEE Conference on Intelligent Transportation Systems (ITSC 2013)', pp. 1559-1564.

Csikós, A., Varga, I. \& Hangos, K. M. (2014), Motorway control system for the reduction of pollutant concentrations in local rural areas, in 'Proceedings of the 22nd Mediterranean Conference on Control \& Automation', pp. 966-971.

Csikós, A., Varga, I. \& Hangos, K. M. (2015), 'Modeling of the dispersion of motorway traffic emission for control purposes', Transportation Research Part C: Emerging Technologies 58, 598-616.

Fontes, T., Pereira, S. R., Fernandes, P., Bandeira, J. M. \& Coelho, M. C. (2015), 'How to combine different microsimulation tools to assess the environmental impacts of road traffic? lessons and directions', Transportation Research Part D: Transport and Environment 34, 293306.

Franklin, G. F., Powell, J. D. \& Emami-Naeini, A. (2002.), Feedback Control of Dynamic Systems., 4th edn, Upper Saddle River, NJ: Prentice Hall, 2002.

Goatin, P., Gottlich, S. \& Kolb, O. (2016), 'Speed limit and ramp meter control for traffic flow networks', Engineering Optimization 48(7), 1121-1144.

URL: http://dx.doi.org/10.1080/0305215X.2015.1097099

Gokhale, S. (2012), 'Impacts of traffic-flows on vehicular-exhaust emissions at traffic junctions', Transportation Research Part D: Transport and Environment 17(1), 21-27.

Gori, S., Spada, S. L., Mannini, L. \& Nigro, M. (2015), 'Emission dynamic meso-simulation model to evaluate traffic strategies in congested urban networks', IET Intelligent Transport Systems 9(3), 333-342.

Grune, L. \& Pannek, J. (2011), Nonlinear Model Predictive Control: Theory and Algorithms, Springer.

Hangos, K. M. \& Cameron, I. T. (2001), Process Modelling and Model Analysis, Academic Press.

Hegyi, A., Schutter, B. D., Hellendoorn, H. \& van den Boom, T. (2002), Optimal coordination of ramp metering and variable speed control-an mpc approach, in 'Proceedings of the American Control Conference', pp. 3600-3605.

Jazcilevich, A., Vázquez, J. M. M., Ramírez, P. L. \& Pérez, I. R. (2015), 'Economicenvironmental analysis of traffic-calming devices', Transportation Research Part D: Transport and Environment 36, 86-95. 
Liu, B., Ghosal, D., Chuah, C. \& Zhang, H. M. (2012), 'Reducing greenhouse effects via fuel consumption-aware variable speed limit (fc-vsl)', IEEE Transactions on Vehicular Technology 61(1), 111-122.

Liu, H., He, K. \& Barth, M. (2011), 'Traffic and emission simulation in China based on statistical methodology', Atmospheric Environment 45, 1154-1161.

Long, J., Chen, J., Szeto, W. Y. \& Shi:, Q. (2016), 'Link-based system optimum dynamic traffic assignment problems with environmental objectives', Transportation Research Part D: Transport and Environment .

Luhar, A. K. \& Patil, R. S. (1989), 'A general finite line-source model for vehicular pollution prediction', Atmospheric Environment 23(3), 555-562.

Ma, X., Jin, J. \& Lei, W. (2014), 'Multi-criteria analysis of optimal signal plans using microscopic traffic models', Transportation Research Part D: Transport and Environment 32, 1-14.

Mahmod, M., Arem, B. V., Pueboobpaphan, R. \& Lange, R. D. (2013), 'Reducing local traffic emissions at urban intersection using its countermeasures', IET Intelligent Transport Systems $7(1), 78-86$.

Ntziachristos, L., Samaras, Z., Eggleston, S., Gorissen, N., Hassel, D., Hickman, A., Joumard, R., Rijkeboer, R., White, L. \& Zierock, L. (2000), Validation of road vehicle and traffic emission models, a review and meta-analysis: Copert iv computer programme to calculate emissions from road transport, methodology and emissions factors (version 2.1), Technical report, European Environment Agency. Tech. Report No. 49.

Office of Transportation and Air Quality (2008), Emission facts, Technical report, United States Environmental Protection Agency (US EPA). Research Report No. EPA420-F-08-025.

Papageorgiou, M., Blosseville, J. M. \& Hadj-Salem, H. (1990), 'Modelling and real-time control of traffic flow on the southern part of boulevard peripherique in paris: Part i: Modelling', Transportation Research A 24, 345-359.

Papageorgiou, M., Kosmatopoulos, E. \& Papamichail, I. (2008), 'Effects of variable speed limits on motorway traffic flow', Transportation Research Record pp 2047, 37-48.

Rakha, H., Ahn, K. \& Trani, A. (2004), 'Development of vt-micro model for estimating hot stabilized light duty vehicle and truck emissions', Transportation Research Part D: Transport and Environment 9(26), 49-74.

Ryu, B. Y., Jung, H. J., Bae, S. H. \& Choi, C. U. (2013), 'Estimation of carbon dioxide emissions per urban center link unit using data collected by the advanced traffic information system in daejeon, korea', Atmospheric Environment 81, 433-442.

Schwede, D. B. (1999), User's guide for the industrial source complex (isc3) dispersion models for use in the multimedia, multipathway and multireceptor risk assessment (3mra) for hwir99. volume 2: Description of model algorithms, Technical report, U.S. Environmental Protection Agency, Office of Solid Waste.

Shorshani, M. F., Seigneur, C., Rehn, L. P., Chanut, H., Pellan, Y., Jaffrezo, J., Charron, A. \& André, M. (2015), 'Atmospheric dispersion modeling near a roadway under calm meteorological conditions', Transportation Research Part D: Transport and Environment 34, 137-154.

Smit, R., Poelman, M. \& Schrijver, J. (2008), 'Improved road traffic emission inventories by adding mean speed distributions', Atmospheric Environment 42, 916-926. 
Smit, R., Smokers, R. \& Rabe, E. (2007), 'A new modelling approach for road traffic emissions: Versit+', Transportation Research Part D: Transport and Environment 12(6), $414-422$.

Szeto, W. Y., Jaber, X. \& Wong, S. C. (2012), 'Road network equilibrium approaches to environmental sustainability', Transport Reviews 32(4), 491-518.

URL: https://doi.org/10.1080/01441647.2012.690000

Yang, H., Wang, X. \& Yin, Y. (2012), 'The impact of speed limits on traffic equilibrium and system performance in networks', Transportation Research Part B: Methodological 46, 12951307.

Yuan, K., Knoop, V. L. \& Hoogendoorn, S. P. (2017), 'A kinematic wave model in lagrangian coordinates incorporating capacity drop: Application to homogeneous road stretches and discontinuities', Physica A: Statistical Mechanics and its Applications 465, 472 - 485.

Zegeye, S. K., de Schutter, B., Hellendoorn, H. \& Breunesse, E. (2009), 'Reduction of travel times and traffic emissions using model predictive control', American Control Conference .

Zegeye, S. K., Schutter, B. D., Hellendoorn, J. \& Breunesse, E. A. (2011), Reduction of areawide emissions using an efficient model-based traffic control strategy, in '2011 IEEE Forum on Integrated and Sustainable Transportation Systems', pp. 239-244.

Zegeye, S. K., Schutter, B. D., Hellendoorn, J., Breunesse, E. A. \& Hegyi, A. (2013), 'Integrated macroscopic traffic flow, emission, and fuel consumption model for control purposes', Transportation Research Part C: Emerging Technologies 31, 158-171.

Zhu, F., Lo, H. K. \& Lin, H.-Z. (2013), 'Delay and emissions modelling for signalised intersections', Transportmetrica B: Transport Dynamics 1(2), 111-135. 